\title{
An Integrated Approach to Life Cycle Analysis
}

\author{
T. M. Chytka ${ }^{*}$, R. W. Brown ${ }^{\dagger}$, A. T. Shih ${ }^{\ddagger}$ J. D. Reeves ${ }^{\S}$, and J.A. Dempsey ${ }^{* *}$ \\ NASA Langley Research Center, Hampton, Va. 23681
}

Life Cycle Analysis (LCA) is the evaluation of the impacts that design decisions have on a system and provides a framework for identifying and evaluating design benefits and burdens associated with the life cycles of space transportation systems from a "cradle-to-grave" approach. Sometimes called "life cycle assessment", "life cycle approach", or "cradle to grave analysis", it represents a rapidly emerging family of tools and techniques designed to be a decision support methodology and aid in the development of sustainable systems. The implementation of a Life Cycle Analysis can vary and may take many forms; from global system-level uncertainty-centered analysis to the assessment of individualized discriminatory metrics. This paper will focus on a proven LCA methodology developed by the Systems Analysis and Concepts Directorate (SACD) at NASA Langley Research Center to quantify and assess key LCA discriminatory metrics, in particular affordability, reliability, maintainability, and operability. This paper will address issues inherent in Life Cycle Analysis including direct impacts, such as system development cost and crew safety, as well as indirect impacts, which often take the form of coupled metrics (i.e., the cost of system unreliability). Since LCA deals with the analysis of space vehicle system conceptual designs, it is imperative to stress that "the goal of LCA is not to arrive at the answer but, rather, to provide important inputs to a broader strategic planning process, allowing the managers to make risk-informed decisions, and increase the likelihood of meeting mission success criteria."

\section{Nomenclature}

$\begin{array}{lll}\text { ARM } & = & \text { Active Risk Manager } \\ \text { CER } & = & \text { Cost Estimating Relationship } \\ \text { CEV } & = & \text { Crew Exploration Vehicle } \\ \text { CLV } & = & \text { Crew Launch Vehicle } \\ \text { CRM } & = & \text { Continuous Risk Management } \\ \text { DDTE } & = & \text { Design Develop Test \& Evaluate } \\ \text { ESAS } & = & \text { Exploration Systems Architecture Study } \\ \text { ESMD } & = & \text { Exploration Systems Mission Directorate } \\ \text { FMEA } & = & \text { Failure Modes and Effects Analysis } \\ \text { FOM } & = & \text { Figures of Merit } \\ \text { FTA } & = & \text { Fault Tree Analysis } \\ \text { GSE } & = & \text { Ground Support Equipment } \\ \text { LOC } & = & \text { Loss of Crew } \\ \text { LOM } & = & \text { Loss of Mission } \\ \text { LOV } & = & \text { Loss of Vehicle } \\ M T B F & = & \text { Mean Time Between Failure } \\ \text { OCM } & = & \text { Operations Cost Model } \\ \text { PCAT } & = & \text { Phased Capability Advanced Technology Architecture } \\ \text { POD } & = & \text { Point of Departure Architecture }\end{array}$

* Systems Engineer/Life Cycle Analyst, Vehicle Analysis Branch, Mail Stop 451, AIAA Member

${ }^{\dagger}$ Systems Engineer/Reliability and Maintainability Analysis, Mail Stop 451, Vehicle Analysis Branch

‡ Systems Engineer/Reliability Analyst, Vehicle Analysis Branch, Mail Stop 451, AIAA Member

§ Systems Engineer/Life Cycle Analyst, Vehicle Analysis Branch, Mail Stop 451, AIAA Member

** Systems Engineer, Space Mission Analysis Branch, Mail Stop 462

The use of trademarks or names of manufacturers in this report is for accurate reporting and does not constitute an official endorsement, either expressed or implied, of such products or manufacturers by the National Aeronautics and Space Administration.

Copyright $\odot 2006$ by the American Institute of Aeronautics and Astronautics, Inc. No copyright is asserted in the United State under Title 17, U. S. Code. The U. S. Government has a royalty-free license to exercise all rights under the copyright claimed herein for Government purposes. All other rights are reserved by the copyright owner. 


$\begin{array}{lll}\text { PRA } & = & \text { Probabilistic Risk Assessment } \\ \text { PRACA } & = & \text { Problem Reporting and Corrective Action } \\ \text { QRAS } & = & \text { Quantitative Risk Assessment System } \\ \text { RBD } & = & \text { Risk Based Design } \\ \text { RMAT } & = & \text { Reliability Maintainability Analysis Tool } \\ \text { S\&MA } & = & \text { Safety \& Mission Assurance } \\ \text { TFU } & = & \text { Theoretical First Unit } \\ V B S & = & \text { Vehicle Breakdown Structure }\end{array}$

\section{Introduction}

Defining and analyzing space exploration architectures requires an understanding of the decisions that drive the risks and life cycle costs of the associated systems. Decisions that affect the risks and costs involve mission parameters, system performance requirements, technology option selections, human machine interfaces, operational capabilities, and "ility" attributes. Dcision-makers at all levels, particularly program office officials and Agency leaders, are often required to make decisions with incomplete or imperfect information; therefore, it is critical that they are provided information generated through strategic systems analysis that captures the sufficient breadth and acceptable depth of the life cycle of a system.

Managing the development of large, complex systems from a strategic perspective presents unique challenges. As systems grow in scope in terms of operations, time, and budget it becomes increasingly more difficult to integrate analysis at the global level. Complex systems are characterized by the fact that they are typically made up of a number of individual component systems; each of which must be analyzed using detailed models. The over-arching goal of strategic analysis is to integrate these segregated analyses to allow effective decision-making at the system of systems level.

Systems Analysis of complex systems involves a number of interrelated functions. First and foremost, system analysis involves the exploration of a trade-space. Typically, analysis models for component systems evaluate point solutions. That is, each run of a model provides analysis of cost, risk, and/or performance for one potential set of independent variables. Strategic analysis provides a structured framework through which dcision-makers can systematically evaluate the entire trade-space and identify optimal solutions. Typically, this means that strategic analysis tools must have the ability to evaluate a large number of independent cases, across ranges and combinations of independent variables, and to identify trends and system drivers across the results.

A strategic analysis capability must be able to integrate the analysis of three distinctly different realms of evaluation; system performance, system economics, and system risk. This integration is challenging because the component systems that make up a global system, while they may operate independently, typically have multiple interdependencies that control the behavior of the global system. Because of these interdependencies, it is not possible to simply evaluate each component system separately and to then integrate the results. As part of strategic analysis, the global system must be evaluated as a whole in order to accurately predict behavior of the system as various elements change. In addition, the three realms of evaluation themselves are not independent. Results for performance, economics, and risk are interdependent and must be evaluated synergistically. Risk results will impact expected performance and expected cost, cost constraints will impact expected performance and programmatic risk, and performance results will impact cost and risk. Strategic analysis must be able to balance all three areas in order to arrive at a feasible and viable solution.

Analysis conducted at a strategic level typically must extend beyond just the missions themselves. Strategic analysis involves an evaluation of all stages of the program lifecycle (i.e., design, development, testing, deployment, operations, and retirement). Again, the results of analysis of the different lifecycle stages are interrelated. A strategic framework must be capable of taking results from one stage of the lifecycle to drive the analysis for other lifecycle stages. The strategic framework must also embed feedback and iteration loops for a true comprehensive systems study.

Many organizations throughout an Agency, such as major program offices, are broken out into functional hierarchies, generally ranging from level 1 (strategic) to level 3 (tactical). Each level of analysis operates within their own set of programmatic trade space boundaries. Strategic levels of analysis and decision-making set the trade-space limits for tactical levels of analysis and often leverage off of such analyses when setting requirements guidance for lower levels. In turn, lower level analyses then provide data and results back up the chain to the higher levels to support the analysis and decision making process. 
For example, based on requirements derived from the Vision for Space Exploration (VSE), Headquarter directorate offices conduct agency strategic analyses. These analyses consider partnerships and competition and evaluate the impact to the NASA Enterprises. The executing organization is the Exploration Systems Mission Directorate (EMSD) (Level 1). Analyses conducted by or for ESMD involved comparing strategic deployment of existing and future space exploration assets and how the deployment affects development, operations, and life cycle costs. The limits of the trade space are set either by the Administrator or ESMD itself. Example trade space definitions and limitations may include: cost profiles, overall budget, key milestones, key mission parameters, or resource leveling needs. Level 1 provided constraints or direction may also include the "Figures of Merit" or FOMs, which will be used to compare options. The goal of Level 1 strategic analysis is to take these trade space limitations and optimize the overall exploration architecture related to the stated objectives (cost, performance, key FOMs).

Deploying a Life Cycle Analysis strategy in strategic and tactical analyses is critical to evaluating the impacts of design decisions on the system trade spaces. LCA provides a framework for identifying and evaluating design benefits and burdens associated with the life cycle of architecture systems from "cradleto-grave" and helps answer two key questions to any design; “Can I afford it?” and "Is it safe?”.

\section{A. Language of LCA:}

As in all disciplines, it is important to clarify the language and terminology that define an analysis. Life Cycle Analysis is often attributed to performing only cost and reliability evaluations, which is an inadequate representation of the true breadth of this discipline. It is a comprehensive examination, evaluation, and quantification of the most complex drivers to a system.

Two commonly misunderstood areas in the field of LCA are reliability versus risk, and cost versus economic analysis. "Reliability is defined as the probability that a system will perform its intended function for a specified period of time under a given set of conditions"”2. Reliability analysis can be conducted at various levels and phases, including component, system, operation, logistics, etc. Risk, defined in relation to reliability, concerns the combined effect of the probability of occurrence of an undesired event with the consequence of the event. Risk analysis must consider the impact of design assumptions on the intricate balance between safety, performance, cost, and schedule. Uncertainty quantification and assessment is also a form of risk analysis, and is often applied to performance characterizations (e.g., mass sizing) and other life cycle metrics such as cost. A complete Life Cycle Analysis will aggregate component reliabilities to facilitate the estimation of overall system reliability risk.

Cost and economic analysis are two additional commonly misunderstood LCA terms. Cost analysis includes the estimation of design, development, test, and evaluation (DDT\&E) cost, and theoretical first unit (TFU) cost based on vehicle characteristics and engineering complexity factors. Cost analysis in context of a life cycle analysis also considers the facility, production, operational, and programmatic costs. "Cost analyses are based on single estimates or amounts for each of the variable quantities considered."” However, "Business decisions frequently involve investments that must be planned and executed many years before the expected returns will be realized”3. Economic analysis provides the tools and strategies required to perform such planning and program execution activities.

\section{B. The Importance of LCA}

All programs must address three key challenges; continual budget constraints, requirements for accelerated research and development schedules, and demand for reliable and safe products and/or processes. Life Cycle Analysis provides the mechanism to integrate the impacts of these constraining design variables to achieve a feasible solution space. In order to meet these competing demands, it is crucial to include LCA in the very early stages of program development and planning because typically $80 \%$ of costs are committed by decisions made during the initial $20 \%$ of the design process ${ }^{4}$. Additionally, as funding resources continue to become constrained, past methods of designing with an objective function of minimizing dry mass do not result in viable designs thus the philosophies of designing with "cost as an independent variable" or "designing for cost" ${ }^{\natural, 7}$ become more important. The inclusion of LCA creates synergy with all disciplines and works towards an optimal and feasible solution allowing dcision-makers to make informed and robust decisions.

\section{SACD's LCA approach}

Within the conceptual space system design community there are "pockets" of expertise that focus on various elements of LCA; often performing analyses independent of one another. SACD has a unique 
approach to LCA in that there is an integrated team of discipline experts working collaboratively with system architects to assess affordability, reliability, maintainability, and operability impacts of design decisions.

Although each LCA provided by the SACD team is unique to each task at hand, a general approach and framework has been developed to guide an analysis. It begins with defining system characteristics including design reference missions and operational protocols. Identifying related requirements is also imperative since it will help define the solution space. Once the system has been properly defined and bounded, the scope of the analysis can be identified, providing dcision-makers with information commensurate with the current design phase. Discriminatory metrics must be formulated in the form of Figures of Merit (FOMs) which are used to assess the impacts of design variables on the system. The next step consists of modeling the system using conceptual design tools and methods, validating model assumptions in context with the current design, and executing the model(s) to generate quantified results. Based on the mission and performance requirements, system reliability, logistics reliability, and maintainability metrics can be calculated. Meanwhile, development and acquisition cost can be estimated based on the vehicle subsystem weights and other critical performance parameters using cost estimating relationships. From the reliability and maintainability assessment, operation and support costs are derived. Furthermore, simulation techniques can be utilized to model vehicle systems and entire mission architectures for single missions or entire campaigns. These simulations allow the probabilistic capture of system complexities and external constraints, and generate metrics of affordability and risk with appropriate statistical descriptor metrics. Using the model output results, the trade space can be explored to alleviate constraining parameters and improve designs consistent with the FOMs. Since the trade space is not fully explored in a single iteration, the life cycle analysis process is iterated synergistically with system architects to mitigate adverse consequences to the system. The results of LCA enable sound decisions to be made concerning research and development portfolios in addition to identifying the best system concepts to pursue.

\section{LCA Application:}

SACD has a proven heritage of providing unbiased life cycle analysis support for a wide range of space systems spanning in-space manned exploration vehicles, robotic science platforms, to both reusable and expendable launch vehicles. A recent LCA activity will be presented in this paper which illustrates the intricacies of Life Cycle analysis.

\section{Language of LCA}

Designing a launch vehicle to have minimal maintenance burden has the greatest potential for cost savings of all of the mission assurance disciplines. Maintainability has a wide range of impacts on such areas as mission facilities, Ground Support Equipment (GSE), system hardware, and ground operations. Unfortunately, the breadth of the subject area makes it impossible to cover maintainability adequately within this paper.

Maintainability is an attribute of a vehicle design that is based on the ease of refurbishing, integrating, servicing, and repairing a vehicle. Maintainability is measured in terms of the resources required for processing the vehicle, including the supplies, manpower, time, and cost, as well as facility and GSE requirements. Because of the differences in launch systems, such as expendable and reusable vehicles, the precise definition of maintainability is dependent on the vehicle requirements.

Maintainability generally impacts costs in two major ways; scheduled maintenance actions and unscheduled maintenance actions. Scheduled maintenance consists of all planned operations. This includes all ground operations required to refurbish, integrate and process a vehicle for launch. It also includes periodic maintenance where components are refurbished or replaced on a scheduled basis. Unscheduled maintenance includes the analysis and repair of unexpected failures of vehicle, facility, and GSE during ground processing that impact the progress toward a timely launch.

Scheduled and unscheduled maintenance impact costs in different ways. Scheduled maintenance costs are simply the cost of doing business and are considered operating costs. Although unscheduled maintenance costs can also be considered operating costs, they are more closely aligned with the cost of unreliability since they are unplanned and unwanted in nature. Just as there is a cost of unreliability associated with when a component fails in flight leading to vehicle loss, there is also a cost of failure when 
a component fails on the ground. This generally takes the form of the repair cost of the component, which impacts the schedule and available resources.

While ground and flight failures contribute to the costs of unreliability, reliability as it applies to maintenance is different from traditional reliability (defined here as operational reliability). Maintenance reliability considers the reliability of any system, sub-system, or component that if it fails, it must be repaired before the next flight. This is different from operational reliability, which concentrates on failures that impact mission results (e.g., loss of vehicle (LOV)). The impact of this definition varies between expendable and reusable vehicles since reusable vehicles can have in-flight failures that do not result in loss of vehicle and must be repaired prior to subsequent missions.

Maintainability also impacts costs that are incurred due to the failure to meet a specified launch date or window. These costs are dependent on the variability of the launch process and are related to availability and probability of launch metrics. For example, additional costs are incurred if you miss a launch window (weather delay, mechanical delay, etc.) which creates added labor hours to service and safe the vehicle while it awaits the next launch opportunity. Since processing times can be described using statistical distributions, the probability of launch on or a before a specific date can be predicted with a certain level of confidence. It is important to note that the probability of launch is not constant throughout vehicle processing, thus the probability of a timely launch should be given for a specific milestone in the process. This probability is based solely on launch processing and should be modified to include other considerations such as weather.

"Reliability is defined as the probability that a system will perform its intended function for a specified period of time under a given set of conditions" ${ }^{\text {. }}$. Reliability analysis can be conducted at various levels of fidelity and design phases, including component, system, operation, logistics, etc. The result of reliability analysis is typically a numeric value reflecting the design reliability of an engineering system that has implications on the operational reliability expressed in terms of loss of mission (LOM), loss of vehicle (LOV), and loss of crew (LOC) metrics. Reliability risk, on the other hand, has three defining characteristics: (1) it is a definable event. (2) There is a probability (or likelihood) the event will happen. (3) There is an impact (or consequence) should the event take place. Therefore, reliability risk concerns the combined effect of the probability of occurrence of an event with the consequence of the event. Risk analysis must consider the impact of design assumptions on the intricate balance between safety, performance (or technical), cost, and schedule.

On a broader perspective, risks can be divided into two categories; internal or external risks. Internal risks are those that are somewhat within project control, such as safety, performance, cost, and schedule risks, whereas external risks are those that are generally beyond program control, such as unpredictable natural disasters, government regulations, changes in inflation, interest rates, etc. Risk analysis is complex and requires diligence to identify, quantify, control and mitigate the effects or consequences on a program. Therefore a strong risk management strategy is essential in program planning.

Life Cycle Cost Analysis (LCCA) and Economic/Affordability Analysis are terms that are often used interchangeably; however, this is not necessarily an accurate assumption. Life Cycle Cost analysis is a systematic approach in applying economics to determine the best solution for a design over the useful life of the system. There are many approaches to LCCA such as cost of borrowing money, present value, depreciation, discount rates, etc. These principles are applicable to the cost elements within a system architecture (DDT\&E, TFU, operations, etc.) Affordability analysis uses the outputs of a LCCA to apply investment strategies over the life cycle of a system such as batch buy philosophies, reserve strategies, learning curves, development cycles, etc. Affordability analysis provides the strategic insight into investment strategies while costing provides the tactical inputs into affordability analysis.

\section{The Importance of LCA}

Quite simply, NASA's charter is to Explore, Discover and Seek to Understand, endeavors which are predicated on vast capital investment and human vulnerability. For this reason, NASA must impose high standards on safety, reliability, cost and schedule control while achieving missions with cutting-edge technology ${ }^{8}$.

NASA, as well as many other commercial and governmental organizations, faces increasing challenges as budgetary constraints intensify in research and development for aerospace and technical development. To respond to these issues, NASA has focused much attention on evaluating impacts of design decisions on the delicate balance between affordability, performance and safety. Affordability analysis provides key 
insights into how to structure investment strategies to meet mission objectives within constrained budgets provides the cost benefit of implementing safety enhancements to a baseline design and allows for thorough exploration of the risk/reliability trade space to fit within acceptable fiduciary limits.

Affordability analysis begins with the determining cost elements within a system architecture. Development costs include the design (initial planning, feasibility studies, and trade space analysis), development, test and evaluation of a prototype system. Acquisition costs consist of production of operational units including the cost of plant start up, fabrication and processing, manufacturing engineering, production control, etc. Operations costs are related to the activities required to operate the system and are often referred to as "bricks and mortar" or "roads to commodes"; it is the infrastructure of the system and all supporting activities. Affordability analysis is important to understanding the key drivers of each of these cost elements and how the interdependencies of these elements impact the affordability of the system.

The safety of the overall system depends on the reliability of the systems ${ }^{9}$. The reliability of a product is strongly influenced by design decisions. Deficiency in design affects the product, and is progressively more expensive and difficult to correct at later stages of development. NASA's exploration initiative demands the use of high performance systems that require tested and matured technologies with greater confidence in reliability predictions. Increased reliability is generally achieved at the expense of increased DDT\&E and production costs. Nevertheless, higher reliability should lower the cost of operation and maintenance due to decreased unscheduled maintenance actions and the reduction of system failures. This is supported by the data which asserts that approximately $80 \%$ of system costs are committed within the initial $20 \%$ of design definition. Therefore, system reliability analysis and design trade studies should be conducted in the conceptual design phase, providing guidance to set a realistic reliability expectation that balances safety, performance, cost and schedule. As the design matures the system design reliability should meet the set reliability goal.

To leverage lessons learned and reduce opportunities for risk, NASA has embraced the philosophy of continuous risk management ${ }^{10}(\mathrm{CRM})$, which is a mechanism to identify, analyze, plan, track, control, document and communicate the risks throughout the project lifetime. The six-step CRM process is a strategy for safety, technical, cost and schedule risk management that seeks to capture the interactions of these elements and mitigate confounding consequences. Risk can be categorized as known risks and unknown risks. As expected, known risks are easier to identify and quantify while unknown risk are often accounted for through risk margins. Whatever the risk categorization, it is imperative to document risk in a risk management plan. Risk analysis, qualitative or quantitative, is a process itself within the greater risk management process. The objective of risk analysis is to lower the risks and reduce the uncertainty associated with risks. Perhaps the most difficult aspect of analyzing a risk is to determine the notional rating or actual numerical value on the event probability. A quantitative risk analysis is definitely preferable, because quantifying the risk's probability affords the opportunity to use more tools to assess the risk, and allows the appropriate degree of planning and mitigating actions to be taken to meet the constraints of work scope, cost and schedule, leading to mission success.

Maintainability affects all of the main elements of a design trade. Its greatest impact however is on affordability. The impact on cost is like the old adage "pay me now, or pay me later". For a system architect this means that you can either design in maintainability with higher development costs, or pay higher operations costs later. Experience has shown that operations cost are many times higher than development costs over the course of a system's life cycle. Unfortunately, project development budget limitations tend to shift costs to later in the life cycle, leading to less than optimal solutions. Assessing the delicate balance between development costs to operations impacts is a fundamental role of LCA.

\section{LCA Methods and Tools}

The discipline of Life Cycle Analysis is plagued with incomplete and imprecise data. In affordability analysis, you may be able to locate analogous data comparable to your system of interest but you have little insight into the "context" of that data. Much of the space mission relevant cost data mined to date does not include some very fundamental information; accounting assumptions, reserve or margin strategies or planned versus actual information. Without insight into what elements compose a cost value of a comparable system, it is difficult to predict the cost of a system of interest. Reliability and maintainability suffer from the same constraint; sparse relevant historical data. Without sound data as a starting point, it is very difficult to build solid prediction models for systems analysis. 
Much of the tools developed for Life Cycle Analysis have been tailored for specific types of systems. One such tool is used for maintenance burden prediction and was developed for reusable launch vehicles. Douglas Morris and Nancy White of NASA's Langley Research Center, and Dr. Charles E. Ebeling of the University of Dayton developed the Reliability and Maintainability Analysis Tool (RMAT). RMAT was developed to estimate the maintainability requirements for next generation reusable launch vehicles. At the heart of the analysis tool is a set of maintainability estimating relationships that relate the vehicle subsystems to the level of maintenance required in the form of spare part counts, workforce estimates, and maintenance actions. Dr. Ebeling developed these relationships based on maintenance data from the Space Shuttle and United States Air Force military aircraft. This provides a maintainability estimate for reusable launch vehicles based on the current behavior of the Shuttle to the ideal (relatively) behavior of military aircraft. For a new launch vehicle, interpolating between the Shuttle and aircraft data points generates maintainability characteristics of each subsystem. The characteristics of each subsystem are then totaled to get a maintenance burden estimate for the entire vehicle.

To provide support for the new Crew Launch Vehicle (CLV) SACD/LCA team will implement a twopronged strategy. First, to develop a maintainability analysis process that provides timely response to the exploration program requirements. Second, to create a basis for the development of a general-purpose tool. The new methodology will be based on a bottoms-up approach to maintainability analysis that is similar to the approach used in reliability analysis.

To support this approach the LCA team is reviving a program called the Conceptual Design Estimating Tool for Reliability and Maintainability, or CoDETRaM. CoDETRaM is based on the Space Shuttle Problem Reporting and Corrective Action (PRACA) database that covers Shuttle flights from STS-51 to STS-90. The database contains the data from approximately 125,000 PRACA reports from the orbiter, external tank, and solid rocket boosters. Data fields include the report title, part number, where the problem occurred during processing, the cause of the problem, and the corrective action taken.

The purpose of CoDETRaM is to provide a user interface to the PRACA records. To support a bottoms-up approach, the records are being sorted using a Vehicle Breakdown Structure (VBS) that assigns each record to a specific system, sub-system and component. The user will be able to select a component of interest and receive a statistical profile on the number of maintenance actions per processing flow. In the future this profile will be expanded to include problem causes, where the problem is discovered, corrective actions, resource requirements, and estimated repair times.

In order to further understand the maintainability impacts on future systems, a Discrete Event Simulation (DES) model is being developed to simulate the ground flow of launch systems. Each event in the model will represent a step in processing the vehicle. The timing of the events can be estimated from historical launch systems or from the expert judgment of launch site personnel. The DES model will also include all of the major resources required for ground processing in the form of facilities, GSE, manpower and vehicle systems.

CoDETRaM data will be introduced into the events in the DES model based on the vehicle components that are affected by each event. Based on components' statistical profiles for each event a probability of problem occurrence can be determined and matched with the appropriate corrective action. Each corrective action will add to the system's demand for resources.

Reusable vehicles must also consider the probability of in-flight equipment failure that does not result in a Loss of Vehicle (LOV) event. This requires that the operational model be expanded to include flight operations. Unlike reliability models that are only concerned with mission, vehicle, and crew losses; the maintainability model must consider the duty cycles of components and their Mean Time Between Failure (MTBF). Each in-flight failure must be accounted for and added as an unscheduled maintenance action during refurbishment, and treated similar to a problem during vehicle integration.

The working model of ground and flight operations can then act as a tool to the decision maker to trade vehicle design, workforce, and support options. It can compare competing options to compare support requirements, and when used in conjunction with Life Cycle Cost Analysis can give the decision maker estimates of the long-term effects of design changes at any point in the design cycle. This will require the designer to look beyond the vehicle and to consider the impacts that the design will have on facilities, GSE, manpower, and sparing requirements.

There are many techniques, software tools and databases available to perform reliability/risk analysis for spacecraft, including qualitative and quantitative methods at different levels of fidelity throughout a project life cycle. Effective reliability predictions should be reviewed within the concept phase because the goal of reliability analyses is to improve the reliability of proposed designs by evaluating the reliability of 
potential system configurations. The SACD LCA team has the capability of performing reliability/risk analysis with various tools from MS-Excel-based analysis to reliability/risk-specific software.

A popularly used and effective reliability analysis method is failure mode and effects analysis (FMEA). FMEA is a 'bottoms-up' method that considers each mode of failure of every component of a system and then identifies the effects on system operation and the overall mission (LOM, LOV, and LOC). Functional and hardware FMEAs have two basic approaches, described in MIL-STD-1629. FMEA can be prepared using MS-Excel for easy editing and updating. FMEA worksheets can be compiled to identify the safety, or critical failure modes and effects, and make recommendations for design improvements for future analysis. FMEA can be extended by incorporating criticality analysis, called FMECA, aiming to rank each potential failure mode according to the combined effect of its severity classification and probability of failure. FMEA/FMECA has gained wide acceptance by the aerospace and the military industries, as well as NASA. In contrast to FMEA, fault tree analysis (FTA) is a 'top-down' method, a systematic and deductive approach of determining the probability of occurrence of an undesirable event (top event) based on a set of constituent lower level events. The events from sub-system and component are connected by logic diagram with "AND" and "OR" gates in a tree-like structure. FTA shows how and why an undesirable event could occur, therefore, captures the hazards, which have potential impacts to more than one sub-system or component. The Safety and Mission Assurance (S\&MA) community frequently adopts the FTA as a hazard analysis tool to produce a list of potential safety hazards. Additionally, given the component failure probability, FTA has been widely used to quantify the probabilities of occurrence of undesirable events leading to LOM, LOV, and LOC in probabilistic risk assessment (PRA).

A digraph model is a graphical model of a system, comprising nodes and edges in a network structure to illustrate the fault propagation paths through a system. Each node represents a failure mode, whereas each directed edge represents a connection through which the occurrence of a failure at one node can flow through the system to cause failure at another node. The logic gates such as "AND" and "OR" are used to describe the system and model correlated failures as in FTA. The model can be constructed from a system schematic diagram augmented with knowledge about the system design and operation, modes of failure and effects (from FMEA), and failure rate of components. Both fault tree analysis and digraph models can be used for system reliability analysis, however, digraphs allow cycles and feed back loops which make it attractive for dynamic system ${ }^{12}$. Through the use of graph theory, the digraph analysis is an aid in determining fault propagation, fault tolerance, and reliability in large, complex, interconnected systems.

The current CLV risk-based design (RBD) activities include the fault propagation logic modeling through the use of the NASA Failure Environment Analysis System-MSFC (FEAS-M) software ${ }^{13}$ based on the digraph method. To support RBD logic modeling task, the SACD LCA team developed the logic model for CLV upper stage (US) main propulsion system (MPS) by interfacing with the RBD FMEA working group and system design team. The mission critical MPS failure modes, causes, and mechanisms were captured in the logic model. The failure rate data are from the existing Space Shuttle PRA v2.1 ${ }^{14}$. Figure 1 depicts a partial MPS fault propagation logic model concerning the failure mode of propellant tank rupture for a reference mission. The MPS logic model was used to predict MPS reliability which was then integrated into whole CLV reliability logic model to obtain CLV reliability predictions and allocations to LOM risk. The intent of integrated CLV reliability assessment is to validate the proposed CLV system reliability requirement, and to provide a reference to the future design trade studies and failure tolerance sensitivity analyses. 


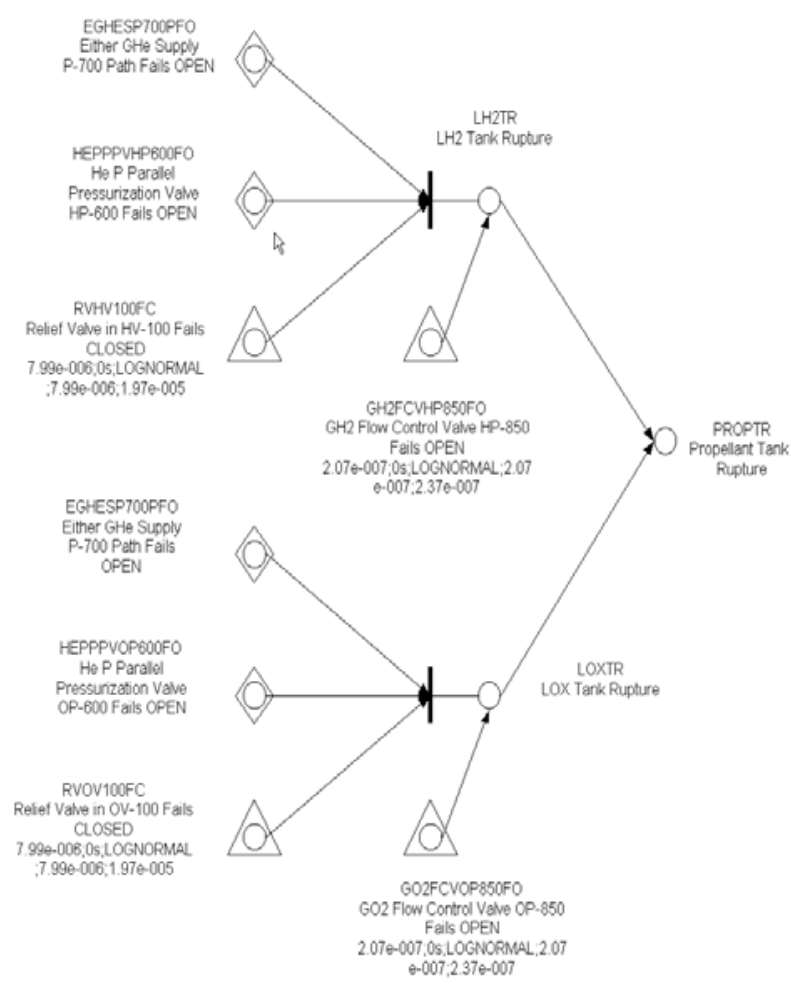

Figure 1: Partial Logic Model for CLV Main Propulsion System using FEAS-M.

In addition to the reliability prediction, probabilistic risk assessment (PRA) has emerged as a popular analysis technique, especially after the Space Shuttle Challenger tragedy in $1986^{14,15,16}$, to evaluate safety risks associated with every life-cycle phase of a complex spacecraft system. Reliability analysis is implemented in PRA by using Boolean logic methods for model development and by statistical or probabilistic methods for quantification portion of the analysis. PRA software (e.g., QRAS $^{17}$ and SAPHIRE ${ }^{18}$ ) not only includes reliability analysis tool, such as FTA, but also has the capability of event tree analysis, common cause failure analysis, master logic diagram, and event sequence diagram (ESD). The results of PRA enable the decision-makers to compare and evaluate the different system designs and operations, and to wisely manage the mission risk (LOM, LOV, and LOC) in a declining budget environment.

After the nation's Vision for Space Exploration initiative was established in January of 2004, NASA embarked on a robust space exploration program. In an independent study, the SACD LCA team was tasked to perform a preliminary PRA on a Point-of-Departure (POD) architecture employing medium lift vehicles for a quadruple launch and quadruple rendezvous mission operation. POD PRA was conducted and a Quantitative Risk Assessment System (QRAS) model was developed ${ }^{19}$ is the screen-capture of QRAS qualitative and quantitative results for the POD architecture. Figure 2(a) shows the considered failures in the mission profile, which is essentially a flowchart from a given initiating event (IE), with paths leading to different end states. The "down" branch is considered to be a "failure"; the end state of a scenario is typically, LOM, LOV, LOC, or any combination thereof. The table in Figure 2(b) provides a ranked list of failure contributions to risk by the failure modes/initiating events for end state of loss of crew and mission.

Classic PRA tools assess potential end states and the impacts related to LOM, LOV, and LOC in the category of safety risk and performance risk, but not the cost and schedule risks that are deemed project risks. NASA's continuous risk management process ${ }^{10}$ employs $5 \times 5$ risk scoring scheme to rate the event probability and event impact at very low, low, moderate, high and very high levels in the area of safety risk, performance, cost and schedule. To facilitate the CRM process, Active Risk Manager ${ }^{20}$ (ARM), a webbased software, has been sanctioned by NASA Exploration Systems Mission Directorate (ESMD) to Implement the risk management of CLV and Crew Exploration Vehicle (CEV) by project teams across NASA centers at different geographical sites. 


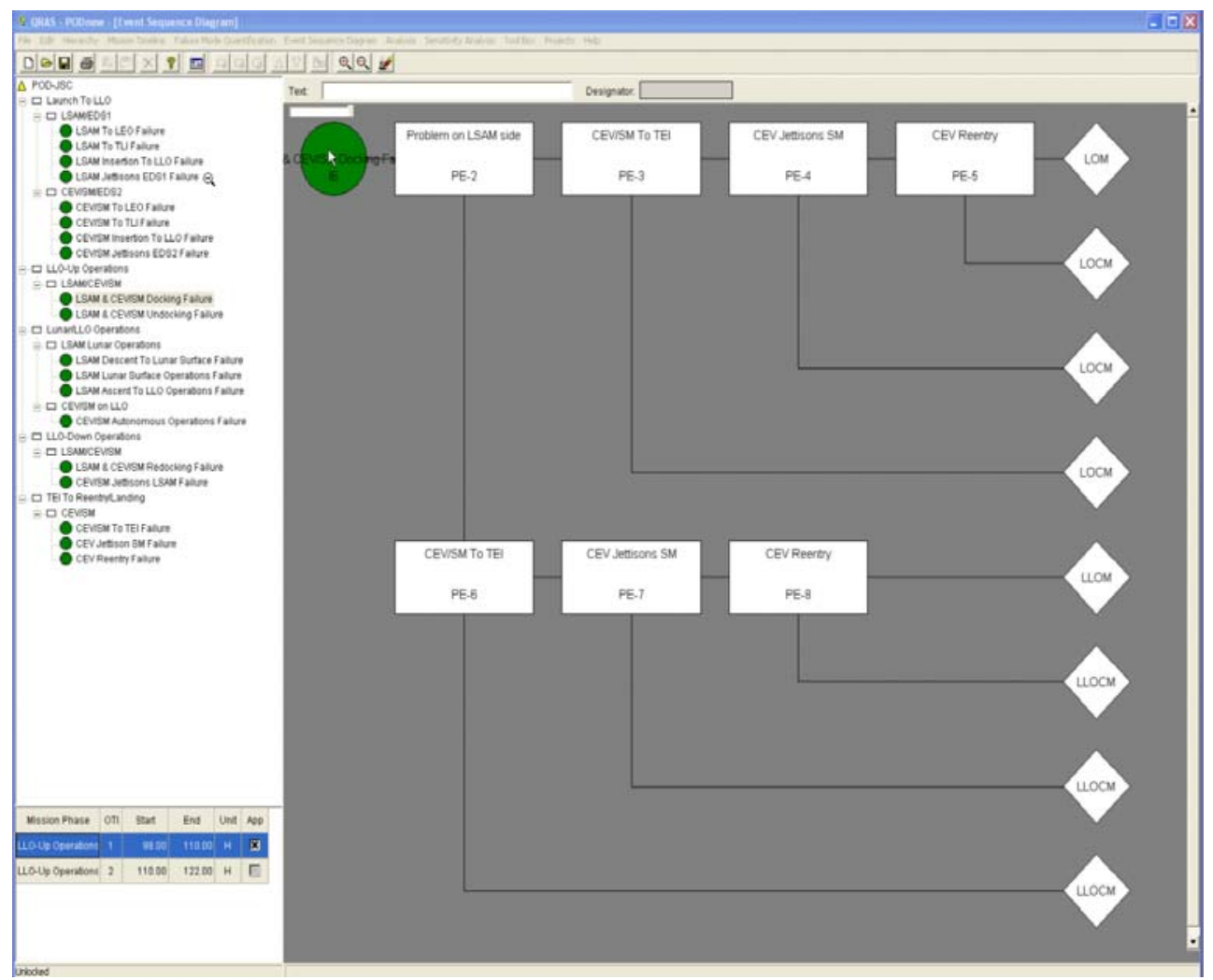

(a) Event Sequence Diagram

\begin{tabular}{|c|c|c|c|c|c|}
\hline 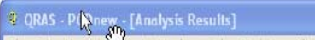 & & 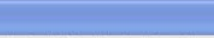 & & & - [ \\
\hline 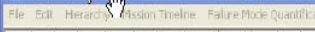 & 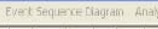 & 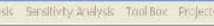 & $x \rightarrow$ Help & & \\
\hline 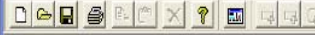 & 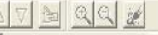 & & & & \\
\hline$\triangle P O D \cdot J S C$ & euarnthitive Result Total & Suantififitie Res suli Ranking | Even Treo & & & \\
\hline $\begin{array}{l}\text { E. L LSAMEDS1 } \\
\text { S LAN TO LEO F Falure }\end{array}$ & Anaysis: New/Anal/si & S5-Run by Phase: Earth To $\llcorner O \longrightarrow \longrightarrow$ & OToEath-Pointes & ntete ony & \\
\hline 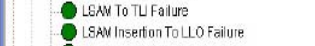 & & & & & \\
\hline $\begin{array}{l}\text { LSAM Jettsons EESI Falurer } \\
\text { 口 CEVIVWEDS2 }\end{array}$ & Selectied Level POO-J & & & & \\
\hline 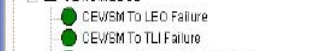 & Select End Staier $\quad$ LO & $\nabla$ & & & Doatale. . \\
\hline 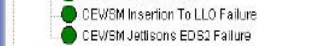 & & & & & \\
\hline 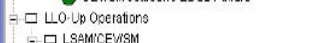 & C Ranking by 9enaric & Finh=kility & & & \\
\hline $\begin{array}{l}E \text { 口 LSAMMCEVISM } \\
\text { - LSAM \& CEVISW Docking Fallure }\end{array}$ & - Ranking per lintiatn & Event: Failure Mode & & & Export. \\
\hline - LANM \& CEVISW Undookng Failure & Faliture Motele Ralk & Intilititug Event & |Mreaul Protatisiliy & So of Total Risk & $\hat{\underline{n}}$ \\
\hline 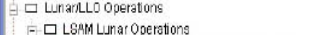 & , 1 & 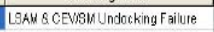 & $6.166=03$ & 2476 & \\
\hline 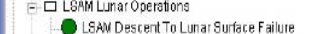 & 2 & LSSM B CEVISIM Redodckng Failure & 6.166003 & 24.76 & \\
\hline 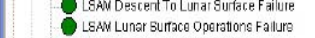 & 3 & LSAM Lunar Surraze Operatonos Fallur & 4.7500003 & 19.07 & \\
\hline L.SNM Aserent To LO O Operations Faliute & 4 & LSAM AserertTo LO Opperalicns Fallu & $1.3522 \mathrm{e} \cdot 3$ & 5.43 & \\
\hline E - CEVISU on LLO & 5 & CEV Reratty Falure & $1.352=-03$ & 543 & \\
\hline - CEVYGM AJtoromous Operations Falure & 8 & CEVJetison SW Falure & $1.352=03$ & 5.43 & \\
\hline$-\square$ LO.DDxn Doerations & 7 & CENSM Lett sons LEAM Fallure & 1.35220 .03 & 5.43 & \\
\hline E L LSAMCEVISM & 8 & LSAM DascentT To Lunar Surrace Fallu & 1.35220 .03 & 5.43 & \\
\hline 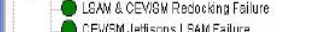 & 9 & CEVSMT T TEIFailure & $7.500=04$ & 301 & \\
\hline 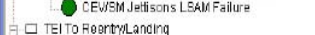 & 10 & CEMSM A A tonomomus ODerafitons Fall. & $4.500=04$ & 1.81 & \\
\hline 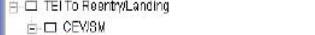 & 11 & LAA I Insatton To LO Fallure & 6.7430 .05 & 0.27 & \\
\hline O CEVSM TO TEI FaluYre & 12 & LSAM \& CEVISM Docking Fallưr & $4.7238-05$ & 0.19 & \\
\hline - CEV Jetision SW Falure & 13 & LSAM Jetisons EDS1 Failure & $7.595=06$ & 0.03 & \\
\hline CEV Reenty Falure & 14 & CEWSM To TIF Falure & $3.463=08$ & 0.00 & \\
\hline & 15 & CEVGM Jett sons EDS2 Felilure & 0.000000 & 0.00 & \\
\hline & 15 & CEVSSM Inserion To LLO Falure & 0.000000 & 0.00 & \\
\hline & 17 & CEWSM Ta LEO Fälure & $0.000=00$ & 000 & \\
\hline & 18 & LSAU TO TLFälure & 0.0000000 & 000 & \\
\hline & 19 & LSAM TO LEO Falure & 0.0000000 & 0.00 & \\
\hline
\end{tabular}

(b) Ranked List Of Failure Modes

Figure 2: Qualitative Risk Assessment System for POD 
Tools and methods for performing cost estimations for space systems is an ever-evolving paradigm. Prior to 1990, no standardized cost estimating tool existed at NASA, instead, numerous spreadsheet models were used. These models relied heavily on volumes of historical data that were searched, analyzed, and inserted into formulas. Additional drawbacks included no formalized training for users, inconsistencies between models, difficulty in showing data traceability, need for engineering judgment, and limited detailed relational analysis capabilities. These models, however, did perform Cost Estimating Relationship (CER) estimates, but provided little more in additional services. As a result, management frequently had to review the findings after cost estimates were generated. In 1990, Marshall Space Flight Centers (MSFC's) Engineering Cost Office visualized a better process using a single model to meet all needs. Within a few years, MSFC, in conjunction with the Air Force, implemented a viable prototype known as the NASA/Air Force Cost Model (NAFCOM). Since then, the NAFCOM has evolved into a cutting-edge cost analysis, modeling, and estimating tool. The NAFCOM consolidates numerous existing cost models and databases used throughout NASA, and brings cost estimating into compliance with today's state-of-the-art software environments. This fully automated software tool employs an easy-to-use spreadsheet environment to predict the cost of space hardware at the subsystem and component levels. The information within the NAFCOM represents the best of the aerospace project data from the Resource Data Storage and Retrieval (REDSTAR) library, NASA's major repository of cost, technical, and programmatic information dating back to the 1960s. The REDSTAR library contains over 22,000 documents and one million pages of information, and maintains a website-based user interface to coordinate these components into a single user-friendly interface. Creating cost estimates within the NAFCOM are based on specific analogy and database averaging techniques. Specific analogy CERs are created by selecting analogous data points from the database within the NAFCOM. The databases average CER represents the average of the data population. To create a specific analogy CER, the user first selects the appropriate database (e.g., manned spacecraft, unmanned orbiting or planetary spacecraft, launch vehicles, liquid rocket engines) and then the appropriate data level (e.g., group, subsystem, component, and unit). Within each data level, the user selects: Group level items (e.g., structures, thermal, and mechanisms; electrical power and distribution; command, control, and data handling); Subsystem level items such as typical aerospace hardware (e.g., thermal communications, attitude control); and Component level data (e.g., batteries, support structure, rate gyros, cabling).After making these selections, the user further refines the CER database by choosing from more than 100 filters within the cost model that relate to the technical and programmatic characteristics of the data points. The available filters are determined by the system and subsystem choices at the data level entry. Once the data levels and filters are applied, the user selects specific programs from a list of missions, enters weights, and applies complexity factors so the NAFCOM can determine the estimated cost.

SACD Estimates operations costs using a tool designed by KT Engineering called Operations Cost Model (OCM). OCM is a top-level operations and support cost model for reusable and expendable launch vehicles. It is predominately a logistics cost model with inputs for general vehicle definition, program inputs, launch operation inputs, flight operations, and facilities. Outputs of the model include ground and mission costs such as facilities cost and engineering support. You may specify up to four flight rates to be evaluated. A typical analysis output is captured in Figure 3.

\begin{tabular}{|c|c|c|c|c|}
\hline \multicolumn{5}{|c|}{ Total Cost vs. Flight Rate } \\
\hline Flight Rate & 1 & 2 & 3 & 4 \\
\hline Program & $\$ 41$ & $\$ 42$ & $\$ 42$ & $\$ 42$ \\
\hline Vehicle & $\$ 103$ & $\$ 104$ & $\$ 105$ & $\$ 105$ \\
\hline Launch & $\$ 215$ & $\$ 219$ & $\$ 222$ & $\$ 224$ \\
\hline Flight & $\$ 12$ & $\$ 13$ & $\$ 13$ & $\$ 14$ \\
\hline SUBTOTAL & $\$ 370$ & $\$ 378$ & $\$ 383$ & $\$ 386$ \\
\hline Wraps & $\$ 0$ & $\$ 0$ & $\$ 0$ & $\$ 0$ \\
\hline TOTAL & $\$ 370$ & $\$ 378$ & $\$ 383$ & $\$ 386$ \\
\hline
\end{tabular}

Figure 3: Operations Cost Model Output 
The benefits of this model is that it provides logistical and operations cost estimates for a life cycle and

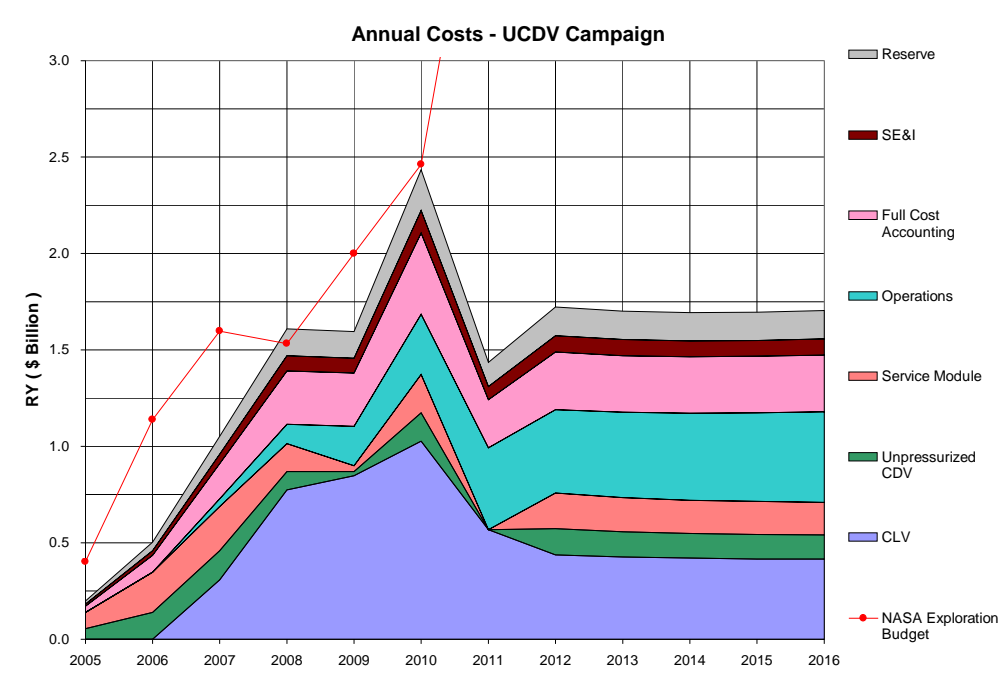

Figure 4: Affordability "Sand Chart" allows investigation of total operations cost sensitivity to various flight rates.

Affordability models are just entering into the NASA suite of Life Cycle Tools. At the moment, they are predominately spreadsheet based models developed by organizations for specific analyses. SACD has developed an affordability model in Microsoft Excel which enables variations in time phasing of money, inflation rates, development schedule perturbations, learning curves, and batch buy strategies. The output of this model is what is often referred to as the "sand

chart"; a graphical representation of the cost of an architecture reflected against a defined budget. Figure 4 illustrates the "sand chart" for the PCAT study.

\section{LCA Applications}

SACD has a proven heritage of providing unbiased life cycle analysis support for a wide range of space systems spanning in-space manned exploration vehicles, robotic science platforms, to both reusable and expendable launch vehicles. Two recent LCA activities include the assessment of an entire manned mission campaign to the lunar surface and a robotic precursor mission to the moon for preparation for later manned missions.

The Phased Capability Advance Technology (PCAT) Architecture Study was an intensive short duration study designed to leverage requirements developed during the Exploration Systems Architecture Study (ESAS) but to challenge those requirements and design configurations to achieve an affordable architecture. The primary objectives of the study were:

- To achieve the exploration mission objectives including staying within cost constraints $\circ$ Spread element development out to stay under the budget curve

- Use existing launch vehicles including a human rated EELV 8mt system;

- Incorporate Modular and Reusable Advanced Concepts and Advanced Technologies

○ Maximize system commonality / Minimize unique systems

- Incorporate system and subsystem reusability

- Evolvable and/or interchangeable systems

- Focus on extensibility of the architecture

- Allow for phased increase of capabilities

- Allow for commercial launch of crew and propellant to low-earth orbit

The system architecture consisted of a Crew Launch and Entry Module (CLEM), a Mission Module (MM), a Rapid Transfer Module (RTM), a Propellant Re-supply Module (PRM), a Lander Module (LM) and a Solar Transfer Module (STM). (See Figure 5 For More Details.) The launch vehicles were an 8mt human rated EELV, and a 25mt cargo delivery EELV. Each of these modules was costed for DDT\&E as well as Production costs. 


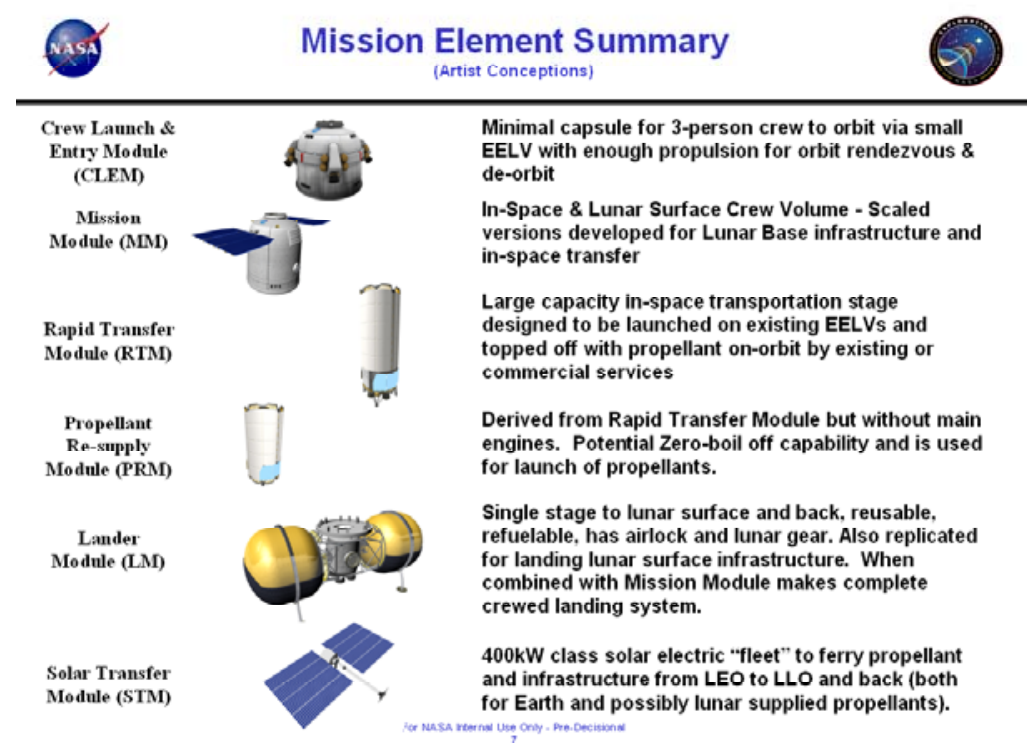

Figure 5: Phased Capability Advanced Technology Architecture

The affordability approach for PCAT was to evaluate the architecture from 2005 through 2025 using our Excel based affordability spreadsheet. A traffic model was incorporated to account for variable yearto-year flight rates, and shown in Figure 6.

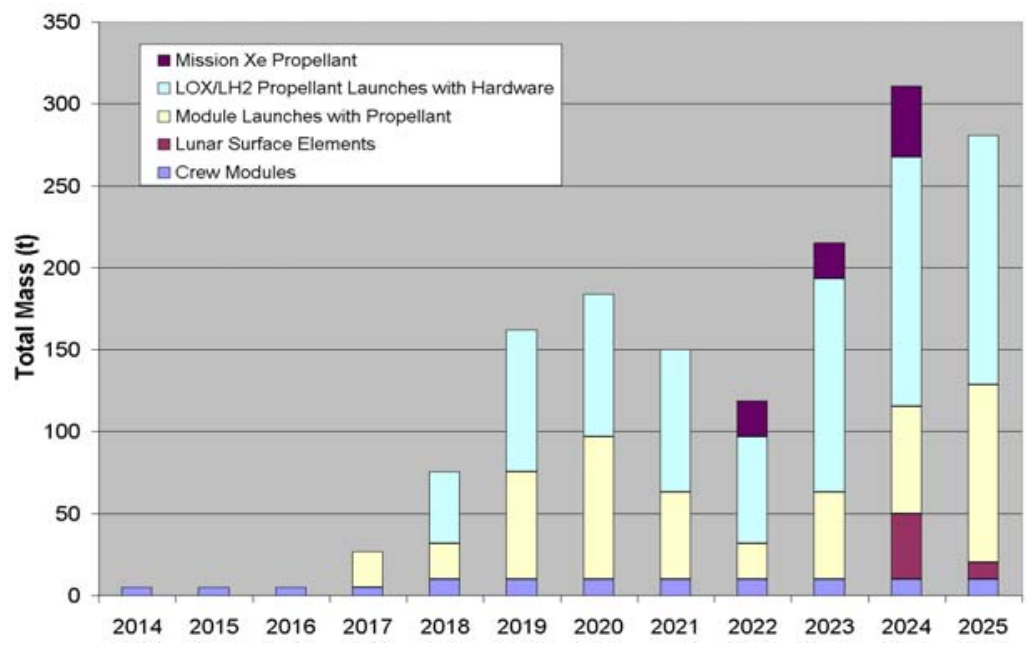

Figure 6: PCAT Traffic Model

To represent a realistic buy profile, production costs were front-loaded then smoothed throughout the remainder of the campaign. Realistic development/production cost distributions were added to the development costing mechanisms in the form of beta distributions to allow for shifting development profiles. Additionally in order to remain within budget, element need dates were allowed to shift based on traffic model assumptions. Due to the intense schedule of this study and constant shifting of design concepts, reliability and risk values were not generated for this study.

The output of this study was a series of affordability curves based on an iterative series of trade space investigations. The beginning baseline assumed a $90 \%$ learning curve on production, a $20 \%$ wrap, and a $25 \%$ reserve rate. This resulted in a life cycle affordability outcome that was very unstable and was not an efficient use of investment dollars. See figure 7. 


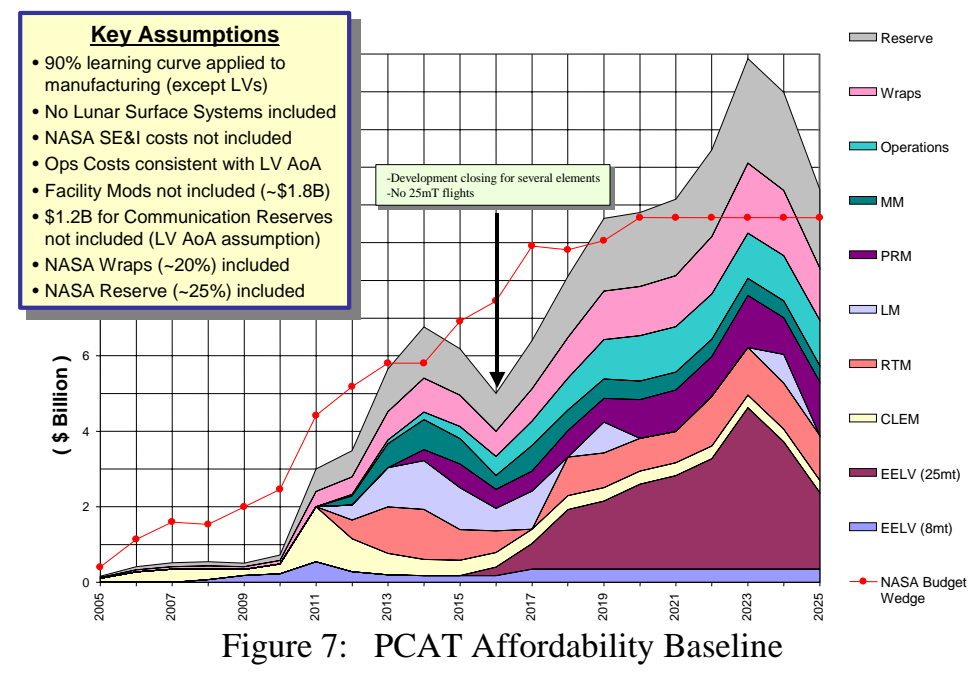

In order to refine and stabilize the funding profile, the $8 \mathrm{mT}$ launch vehicle development start time shifted up by 2 years, a 14 year batch buy paradigm was applied to the $25 \mathrm{mt}$ launch vehicle, the lunar module development start time was accelerated by 3 years and the crew launch and entry module development start time was accelerated by 3 years and development duration was reduced from 9 years to 6

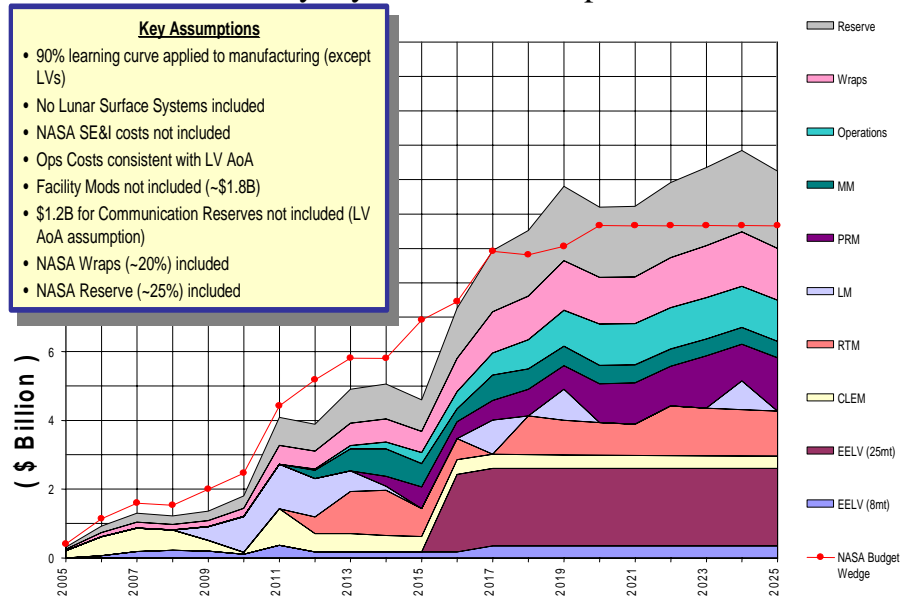

years. This resulted in the affordability profile in Figure 8.

Figure 8: PCAT Affordability Iteration

The outcome of this study indicated that in order for the system elements to fit under the budget curve and the architecture exceeded budget only in reserve status, significant modifications to development schedules and production buy philosophies would need to be employed.

\section{Conclusions}

Life cycle analysis captures all phases of a system, from concept development, to production, to cost of ownership, to retirement. It is a dynamic, statistics-based, highly-complex discipline that provides crucial figures of merit, and encapsulates the most important tenants of any design; affordability and risk. The Life Cycle Analysis Team within the Systems Analysis and Concepts Directorate (SACD) at NASA Langley Research Center strives to provide qualitative and quantitative life cycle analyses to assess the impacts of design decisions on key figures of merit and has a strong heritage of providing information to dcisionmakers that helps drive the system trade space to a feasible solution. Again, "the goal of LCA is not to arrive at the answer but, rather, to provide important inputs to a broader strategic planning process, 
allowing the managers to make risk-informed decisions, and increase the likelihood of meeting mission success criteria."

\section{REFERENCES}

${ }^{1}$ Svoboda, S., "Note on Life Cycle Analysis", website http://www.umich.edu/ nppcpub/resources/compendia/CORPpdfs/CORPlca.pdf

${ }^{2}$ Lewis, E.E., Introduction to Reliability Engineering, Second Edition, John Wiley \& Sons, Inc., N.Y., 1996.

${ }^{3}$ Canada, J.R., Sullivan, W.G., White, J.A., Capital Investment Analysis for Engineering and Management, Second Edition, Prentice Hall, Inc., Upper Saddle River, N.J., 1996.

${ }^{4}$ Dieter, G.E., Engineering Design, Third Edition, McGraw Hill, Boston, Ma., 2000.

${ }^{5}$ NASA Cost Estimating Handbook, 2004.

${ }^{6}$ Wertz, J. and Larson, W.J., Reducing Space Mission Cost, Microcosm Press, El Segundo, Ca., 1996.

${ }^{7}$ Dean, E and Unal, R., "Designing for Cost", Conference of the American Association of Cost Engineers, 1991.

${ }^{8}$ NPR 7120.5C, "NASA Program and Project Management Processes and Requirements", March, 2005.

${ }^{9}$ Fragola, J.R., et al., "Reliability and Crew Safety Assessment for A Solid Rocket Booster/J-2S Launcher", SAICNY05-04-1F.

${ }^{10}$ NPR 8000.4, "Risk Management Procedural Requirements w/Change 1”, April, 2004.

${ }^{11}$ Goldberg, B.E., et al., "System Engineering “Toolbox” for Design-Oriented Engineers”, NASA Reference Publication 1358, December, 1994.

${ }^{12}$ Gantzer, D.J., ”Using a Directed Graph Methodology for Integrated Risk Assessment on Space Station Freedom Program", AIAA-93-4675.

13 "Preliminary FEAS-M Failure Environment Analysis System MSFC”, Version 2.0 Users Guide, September 2003, MSFC Group.

${ }^{14}$ Space Shuttle Probabilistic Risk Assessment. Version 2.1, NASA JSC Shuttle Project, 2004.

${ }^{15}$ Safie, F.M., “An Overview of Quantitative Risk Assessment of Space Shuttle Propulsion Elements”, 4th International Association for Probabilistic Safety Assessment and Management (IAPSAM), September 13-18, 1998, New York.

${ }^{16}$ Fragola, J.R., et al., "Probabilistic Risk Assessment of the Space Shuttle. Phase 3: A Study of the Potential of Losing the Vehicle during Nominal Operation", 1995.

17 “Quantitative Risk Assessment System (QRAS)”, Version 1.7 Users’ Guide, December, 2002.

18 "Systems Analysis Programs for Hands-on Integrated Reliability Evaluations (SAPHIRE)”, Version 7, 1999.

${ }^{19}$ Pate-Cornell, M.E. and Dillon, R.L., "Probabilistic Risk Analysis for the NASA Space Shuttle: a Brief History and Current Work”, Reliability Engineering \& System Safety 74 (3): 345-352, December, 2001.

${ }^{20}$ Active Risk Manager (ARM), Version 3.0, 2006 by Strategic Thought Group. 